\title{
Téenica de Calibração de Sensores Meteorológicos de Temperatura e Umidade Relativa do ar Utilizando um Sensor de Referência
}

\author{
Calibration Technique of Meteorological Sensors for Temperature and Relative Humidity Using a Reference \\ Sensor
}

\author{
Jonathan Willian Zangeski Novais*a; Ana Cristina Hillesheima; Naiara Cristina Fank; ${ }^{\mathrm{a}}$, Louyse Siqueira Varella \\ Oliveira $^{a}$; Naara Soares dos Santos Brito ${ }^{\text {b }}$ Dahiane dos Santos Oliveira Zangeski ${ }^{\mathrm{a}}$; Bárbara Bella Pereira De Oliveira ${ }^{\mathrm{c}}$ \\ Universidade de Cuiabá, Programa de Pós-Graduação Stricto Sensu em Ciências Ambientais. MT, Brasil. \\ bUniversidade de Cuiabá. MT, Brasil. \\ 'Instituto Federal de Mato Grosso. MT, Brasil. \\ *E-mail: jonathan.novais@kroton.com.br
}

\begin{abstract}
Resumo
Em pesquisas ambientais é comum a utilização de mais de um sensor meteorológico para medições, principalmente, para medições espacializadas. Porém pode ocorrer erro na coleta espacializada, quando os sensores são diferentes. Nesse contexto, por meio da regressão linear se pode calibrar as medições dos sensores utilizados em função de um sensor de referência. Assim, este trabalho tem como objetivo apresentar uma técnica de calibração de sensores meteorológicos, focado nas variáveis temperatura do ar e umidade relativa do ar, utilizando um sensor de referência, e fazendo a aplicação posterior em campo. Os sensores foram calibrados em ambiente controlado, em que se podia controlar a temperatura do ar e a umidade relativa do ar, de forma a submeter os sensores a uma amplitude maior de variações, variando de $25{ }^{\circ} \mathrm{C}$ a $45{ }^{\circ} \mathrm{C}$ de temperatura do ar e de $20 \%$ a $60 \%$ de umidade relativa do ar. Os resultados das regressões lineares foram considerados satisfatórios, uma vez que todos os coeficientes angulares e lineares foram considerados significativos e o menor coeficiente de determinação $\left(\mathrm{R}^{2}\right)$ foi de 0,91 . Após a calibração foram feitas medidas meteorológicas no Parque das Águas, Cuiabá-MT, de modo a aplicar as regressões em dados reais. As regressões corrigiram diferenças máximas de $0,9{ }^{\circ} \mathrm{C}$ de temperatura do ar e $8,6 \%$ de umidade relativa do ar. Conclui-se que a metodologia é válida para a calibração de sensores diferentes em relação a um sensor de referência, podendo ser uma estratégia interessante para os pesquisadores, que não possuem diversos sensores calibrados.
\end{abstract}

Palavras-chave: Temperatura do Ar. Umidade Relativa do Ar. Estação Meteorológica. Termohigrômetro.

\begin{abstract}
In environmental research it is common to use more than one meteorological sensor for measurements, mainly for spatialized measurements. However, there may be an error in the spatial collection when the sensors are different. In this context, through linear regression, it is possible to calibrate the measurements of the sensors used in function of a reference sensor. Thus, this work aims to present a calibration technique of meteorological sensors, focused on the variables air temperature and the air relative humidity, using a reference sensor, and making the subsequent application in the field. The sensors were calibrated in a controlled environment, in which air temperature and relative humidity could be controlled, in order to subject the sensors to a greater range of variations, ranging from $25{ }^{\circ} \mathrm{C}$ to $45{ }^{\circ} \mathrm{C}$ and $20 \%$ at $60 \%$ relative humidity. The linear regressions results were considered satisfactory, since all the angular and linear coefficients were considered significant and the lowest determination coefficient $\left(R^{2}\right)$ was 0.91. After calibration, meteorological measurements were made in Parque das Aguas, $C$ Cuiabá-MT, in order to apply the regressions to real data. The regressions corrected maximum differences of $0.9^{\circ} \mathrm{C}$ of air temperature and $8.6 \%$ of relative air humidity. It is concluded that the methodology is valid for the calibration of different sensors in relation to a reference sensor, which can be an interesting strategy for researchers who do not have several calibrated sensors.
\end{abstract}

Keywords: Air Temperature. Relative Humidity. Weather Station. Thermohygrometer.

\section{Introdução}

Em função do alto custo de estações meteorológicas automáticas (TORRES et al., 2015), é comum o uso de sensores de baixo custo, ou associar a utilização destes com sensores de referência, que possuam melhor precisão, principalmente, em locais nos quais não há abrangência da rede meteorológica oficial e em locais remotos (BARATTO et al., 2020).

Para medidas espacializadas, em malha ou em transectos, é comum o uso de mais de um sensor meteorológico, de forma que as medidas sejam mais próximas possíveis temporalmente, uma vez que a radiação solar varia constantemente, alterando assim variáveis como temperatura do ar e do solo, umidade relativa do ar, vento e pressão atmosférica, a utilização de mais de um sensor pode contribuir para minimizar os erros que poderiam existir, caso todas as coletas fossem feitas com apenas um sensor, pois a primeira e última medida teriam diferenças causadas pela variação temporal da radiação solar, e não em decorrência de condições meteorológicas locais instantâneas. Dessa forma, é comum a análise e comparação entre sensores de modelos diferentes, sensores de baixo custo. Essa prática, porém, gera a preocupação da qualidade das medidas geradas, uma vez que sensores com precisões e acurácias diferentes geram resultados diferentes para medidas que, em teoria, deveriam ser iguais.

A necessidade da utilização de sensores de baixo custo é uma ocorrência atual entre pesquisadores. Rojas et al. (2014), 
por exemplo, avaliaram diferentes abrigos meteorológicos para auxiliar o monitoramento climático em países em desenvolvimento. No Brasil, diversos pesquisadores, na área de instrumentação, desenvolvem sensores de baixo custo (DA CUNHA et al. 2001; SOUZA et al. 2015; TORRES et al. 2015), com o objetivo de disponibilizar estas tecnologias para um número maior de usuários.

Nesse sentido, em pesquisas que se utilizam de mais de um sensor ou sensores de baixo custo, por meio de estatística e de um sensor meteorológico de referência se podem minimizar as diferenças de medições entre os sensores, podendo evitar da contratação de laboratórios especializados, uma vez que esse tipo de gasto nem sempre está disponível para todos os pesquisadores.

Este trabalho tem como objetivo apresentar uma técnica de calibração de sensores meteorológicos, focado nas variáveis temperatura do ar e umidade relativa do ar, utilizando um sensor de referência.

\section{Desenvolvimento}

\subsection{Instrumentação meteorológica}

Foram utilizados nesta pesquisa 5 termohigrômetros digitais móveis, para medidas de temperatura do ar (Tar) e umidade relativa do ar (UR), sendo um deles o termohigrômetro de referência denominado como " $R$ ", constando com certificado de calibração de fábrica fornecido pela fornecedora, e o restante denominado como: "A", "B", "C" e "D". O sensor "A", apesar de ser do mesmo modelo do sensor de referência, é um sensor mais antigo, com o certificado de calibração já expirado e os sensores " $B$ ", " $C$ " e "D" são de modelo e fabricantes diferentes. As descrições técnicas e modelos dos sensores seguem abaixo no Quadro 1.

Quadro 1 - Fornecedora, modelo, faixas de medição, acurácia e resolução dos 5 sensores termohigrômetros analisados, para medidas de temperatura do ar (Tar) e umidade relativa do ar (UR), feitas em laboratório no dia 10/09/2020.

\begin{tabular}{|c|c|c|c|c|c|c|c|c|}
\hline Código & Fornecedora & Modelo & $\begin{array}{l}\text { Faixa de } \\
\text { medição } \\
\text { para Tar }\end{array}$ & $\begin{array}{l}\text { Faixa de } \\
\text { medição } \\
\text { para UR }\end{array}$ & $\begin{array}{c}\text { Acurácia da } \\
\text { Tar }\end{array}$ & $\begin{array}{c}\text { Acurácia da } \\
\text { UR }\end{array}$ & $\begin{array}{l}\text { Resolução } \\
\text { da Tar }\end{array}$ & $\begin{array}{c}\text { Resolução } \\
\text { da UR }\end{array}$ \\
\hline $\mathrm{R}$ & NK Company & Kestrel 4500 & $\begin{array}{c}-29^{\circ} \mathrm{C} \text { a } 70 \\
{ }^{\circ} \mathrm{C}\end{array}$ & $5 \%$ a $95 \%$ & $0,5^{\circ} \mathrm{C}$ & $3 \%$ & $0,1^{\circ} \mathrm{C}$ & $0,1 \%$ \\
\hline A & NK Company & Kestrel 4500 & $\begin{array}{c}-29^{\circ} \mathrm{C} \text { a } 70 \\
{ }^{\circ} \mathrm{C}\end{array}$ & $5 \%$ a $95 \%$ & $0,5^{\circ} \mathrm{C}$ & $3 \%$ & $0,1^{\circ} \mathrm{C}$ & $0,1 \%$ \\
\hline B & $\begin{array}{c}\text { Oregon } \\
\text { Scientific }\end{array}$ & THG 312 & $-5^{\circ} \mathrm{C}$ a $50{ }^{\circ} \mathrm{C}$ & $25 \%$ a $95 \%$ & $\pm 1^{\circ} \mathrm{C}$ & $\begin{array}{c}\text { não } \\
\text { informado }\end{array}$ & $0,1^{\circ} \mathrm{C}$ & $\begin{array}{c}\text { não } \\
\text { informado }\end{array}$ \\
\hline $\mathrm{C}$ & $\begin{array}{c}\text { Oregon } \\
\text { Scientific }\end{array}$ & THG 312 & $-5^{\circ} \mathrm{C}$ a $50^{\circ} \mathrm{C}$ & $25 \%$ a $95 \%$ & $\pm 1^{\circ} \mathrm{C}$ & $\begin{array}{c}\text { não } \\
\text { informado }\end{array}$ & $0,1^{\circ} \mathrm{C}$ & $\begin{array}{c}\text { não } \\
\text { informado }\end{array}$ \\
\hline $\mathrm{D}$ & $\begin{array}{c}\text { Oregon } \\
\text { Scientific }\end{array}$ & THG 312 & $-5^{\circ} \mathrm{C}$ a $50^{\circ} \mathrm{C}$ & $25 \%$ a $95 \%$ & $\pm 1^{\circ} \mathrm{C}$ & $\begin{array}{c}\text { não } \\
\text { informado }\end{array}$ & $0,1^{\circ} \mathrm{C}$ & $\begin{array}{c}\text { não } \\
\text { informado }\end{array}$ \\
\hline
\end{tabular}

Fonte: dados da pesquisa.

\subsection{Descrição da calibração}

A calibração foi feita no dia 10/09/2020, em laboratório com ambiente controlado podendo, por meio da utilização de ar-condicionado e umidificador de ar, variar a temperatura do ar e umidade relativa do ar em extremos quentes, frescos, secos e úmidos, sujeitando os sensores aos diversos tipos de situações. Todos os sensores foram postados um ao lado do outro, dispostos horizontalmente e sem a incidência de radiação solar direta sobre esses.

A faixa de temperatura ambiente ocorreu entre $26{ }^{\circ} \mathrm{C}$ e $45,6{ }^{\circ} \mathrm{C}$ e para umidade relativa do ar de $23 \%$ a $60 \%$. Os sensores ficaram expostos às variações em um período de 4 horas, sendo as medidas tomadas de 10 em 10 minutos.

De forma a estabelecer relações matemáticas entre os sensores calibrados e o de referência foi utilizado o método da regressão linear, de forma a estabelecer uma equação de ajuste para cada par de conjuntos de dados (R e A; R e B; R e C; R e D). O termo "Análise de Regressão" define um conjunto vasto de técnicas estatísticas usadas para modelar relações entre variáveis e predizer o valor de uma ou mais variáveis dependentes (ou de resposta), a partir de um conjunto de variáveis independentes (ou preditoras)" (MAROCO, 2007).

De forma a verificar o ajuste do modelo se calculou o coeficiente de determinação $\left(\mathrm{R}^{2}\right)$ da regressão linear, que é o quadrado do coeficiente de correlação amostral r (MARTINS, 2018), sendo uma medida de ajuste de um modelo estatístico linear generalizado, variando de 0 a 1 , sendo 0 nenhum ajuste e 1 o ajuste perfeito.

Testou-se pelo teste de Shapiro-Wilk a normalidade e teste de Levene para homocedasticidade, e ao não atender os critérios se utilizou da estatística não-paramétrica para a comparação de médias, sendo utilizado o teste de MannWhitney para comparar os dados dos sensores antes e depois da calibração. Todos os testes estatísticos foram feitos no Statistical Package for Social Sciences (SPSS), versão 22.0 (IBM Corp, NY, Estados Unidos).

\subsection{Medições de campo}

As coletas ocorreram no Parque das Águas, Figura 1, localizado no município de Cuiabá/MT, em volta da Lagoa Paiaguás. O parque possui espaço para atividades de esporte e lazer, porém boa parte de sua superfície está impermeabilizada, com pouca vegetação existente, inviabilizando a sua utilização 
durante boa parte do dia.

Figura 1 - A) Área total do Parque das Águas, Cuiabá - MT. B) Área de medição focada na região do entorno da Lagoa Paiaguás.

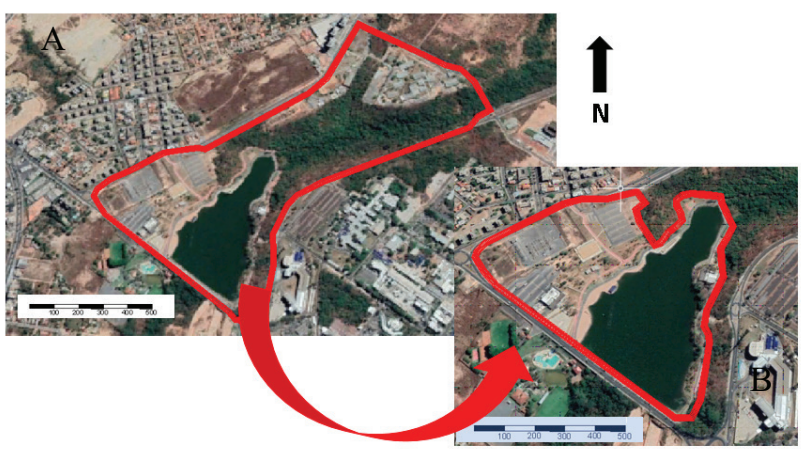

Fonte: Adaptado de Google Earth (2020).

De modo a aplicar as equações geradas foram monitorados na região, em torno da Lagoa Paiaguás, 20 pontos, conforme figura 1-B, pelo método do transecto móvel, em que foram usados os 5 sensores e cada sensor mediu 4 pontos. A coleta ocorreu no dia 10 de fevereiro de 2020, sendo escolhido um dia ensolarado, sem a ocorrência de precipitação no dia. Os horários das medições foram às 8,14 e 20 horas.

\subsection{Discussão}

\subsubsection{Resultados das regressões lineares}

Segue abaixo, na Figura 2, as regressões lineares e os respectivos coeficientes de determinação para a calibração dos sensores referentes às temperaturas do ar.

Figura 2 - Regressão linear e coeficiente de determinação da calibração dos sensores referente a temperaturas do ar.
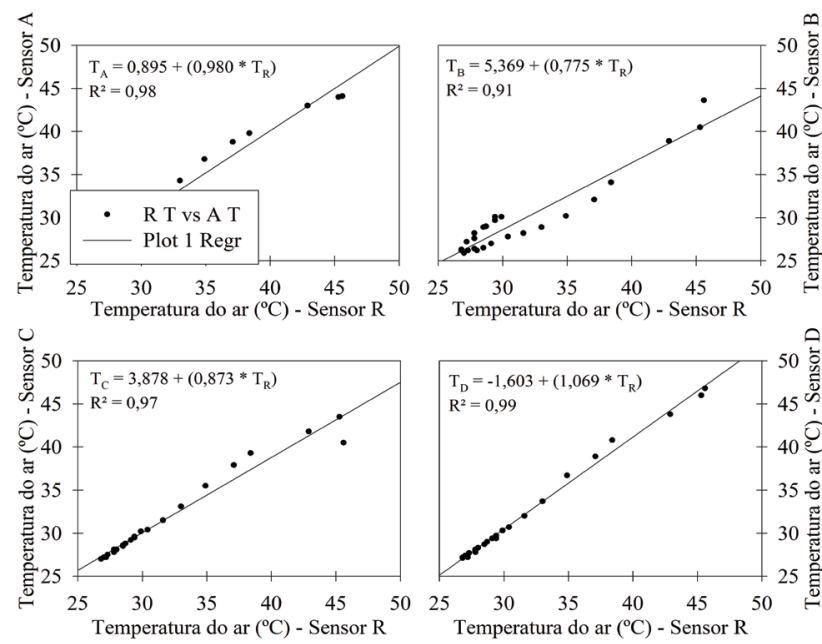

Fonte: Dados da pesquisa.

Analisando os coeficientes de correlação se nota que o menor coeficiente de determinação foi 0,91 , para o sensor "B", indicando um bom ajuste das medições ao modelo. Testou-se a significância de todos os coeficientes lineares e angulares das regressões, sendo todos significativos a um nível de 5\% (p-valor $<0,05)$.
Figura 3 - Regressão linear e coeficiente de determinação da calibração dos sensores referentes à umidade relativa do ar
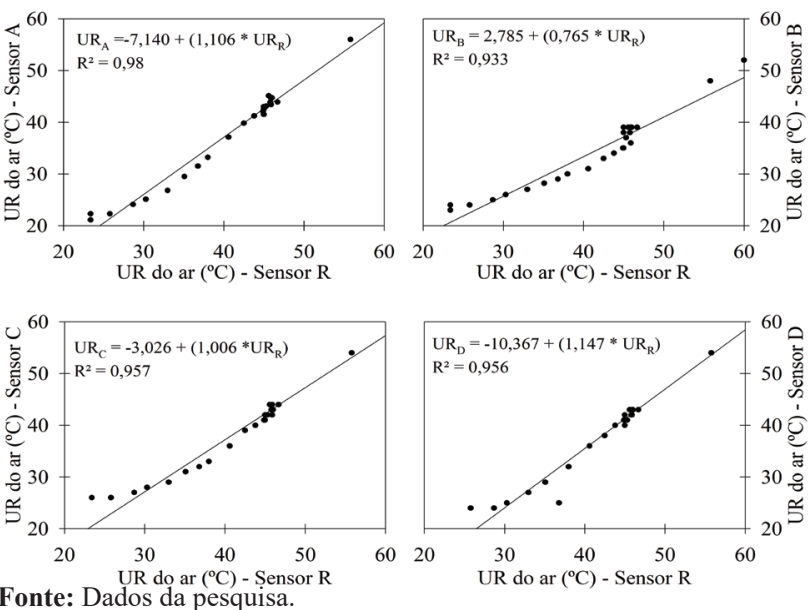

Fonte: Dados da pesquisa.

Os resultados para umidade relativa do ar foram similares aos de temperatura do ar, em que o menor coeficiente de determinação foi de 0,933 , também para o sensor "B". Os coeficientes angulares e lineares das regressões também foram significativos a um nível de 5\% (p-valor $<0,05)$.

\subsubsection{Aplicação em campo}

De forma a aplicar os ajustes em situação real, coletaramse os dados de temperatura do ar e umidade relativa do ar no Parque das Águas, utilizando os 5 sensores, e esses foram comparados (Figura 4).

Figura 4 - Comparação entre as medidas de temperatura do ar para os 20 pontos do transecto do Parque das Águas, em Cuiabá - MT, no dia 10/02/2020, às 8,14 e 20 horas

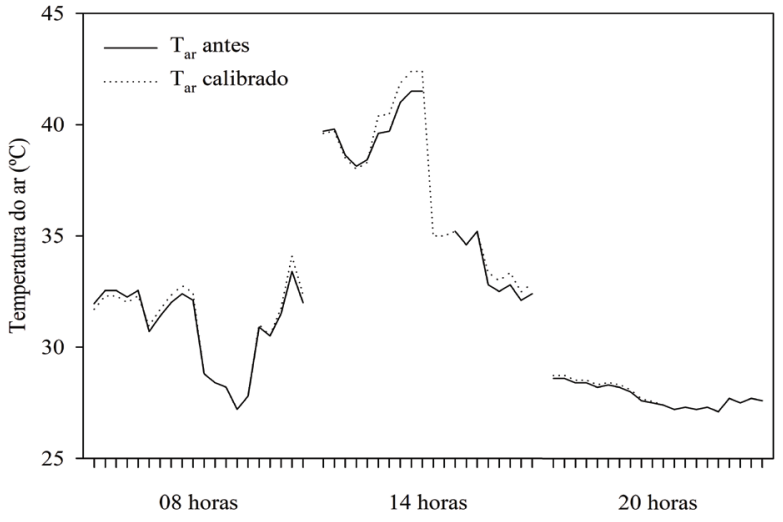

Fonte: Dados da pesquisa.

Observam-se diferenças maiores, no período vespertino, uma vez que em função de altas temperaturas, os sensores tendem a diferir. Já para temperaturas abaixo de $30{ }^{\circ} \mathrm{C}$, os sensores tenderam a medidas mais similares.

Apesar de algumas diferenças gráficas, em que ocorreram diferenças de até $0,9{ }^{\circ} \mathrm{C}$, não ocorreu pelo teste de MannWhitney e diferenças estatísticas significativas para nenhum dos 3 períodos. Isso pode ser explicado pela acurácia similar entre os sensores de temperatura do ar e também em função da 
série calibrada constar com dados do sensor de referência, uma vez que as coletas ocorreram de forma simultânea, utilizando os 5 sensores, e uma faixa de coleta foi feita pelo próprio sensor de referência, podendo ser visualizado na figura 4 nos pontos em que as linhas estão sobrepostas.

Destaca-se a temperatura máxima de $42,4{ }^{\circ} \mathrm{C}$ às 14 horas. Conforme Gartland (2010), superfícies constituídas por asfalto, normalmente, apresentam temperaturas elevadas se comparadas aos outros materiais. Maciel (2014) reitera que esse efeito é explicado pelo baixo valor de albedo desse material, que resulta em grande absorção da energia térmica proveniente da radiação solar. Este ponto é localizado próximo da região de estacionamento, que é acometida por grande incidência de radiação solar em decorrência da ausência de vegetação, contribuindo para o aumento da temperatura do ar que, conforme Novais et al. (2020), o asfalto betuminoso absorve grande parte dessa energia, em função de seu alto calor específico, acumulando e transmitindo para o meio em forma de calor (ondas de infravermelho).

Segue, na Figura 5, a comparação para a umidade relativa do ar para os 20 pontos analisados nos 3 períodos do dia.

Figura 5. Comparação entre as medidas de umidade relativa do ar para os 20 pontos do transecto do Parque das Águas em Cuiabá - MT, no dia 10/02/2020, às 8,14 e 20 horas

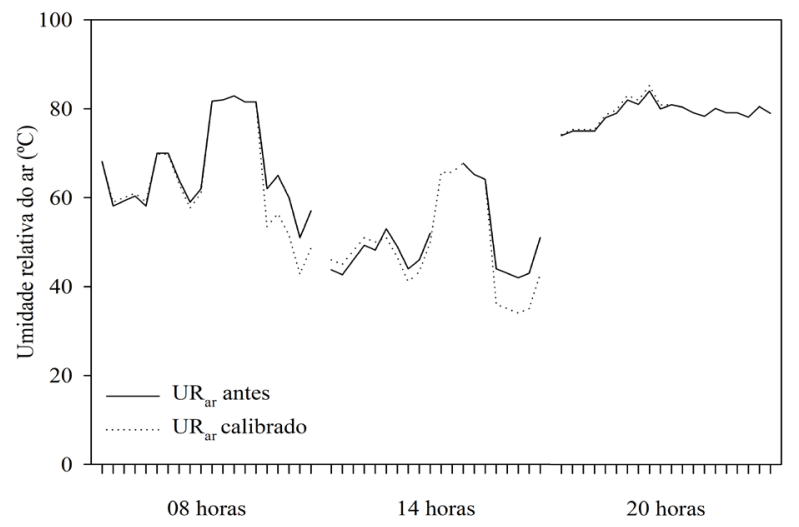

Fonte: Dados da pesquisa.

Observam-se diferenças entre as séries no período matutino e vespertino, principalmente, nos pontos finais de coleta, ocorrendo pontos de até $8,6 \%$ de diferença. No período noturno, em função da ausência de radiação solar e manutenção da temperatura, a amplitude de umidade relativa foi menor entre os pontos e entre as diferenças das séries coletadas. Apesar das diferenças dos pontos finais, também não ocorreram diferenças estatísticas significativas entre as séries antes e depois da calibração.

As variações de umidade relativa do ar em todos os períodos decorrem de diferentes tipos de cobertura e sombreamento encontrados no parque, ocorrendo os maiores valores de umidade relativa do ar nas regiões mais arborizadas do parque.

Constatou-se, também, em ambos os períodos do ano, que os maiores valores de umidade relativa do ar observados correspondem aos menores valores de temperatura do ar e vice-versa, isto ocorre em função do fato de que, quando a temperatura diminui, a quantidade de vapor de água no ar não muda, mas a umidade relativa do ar aumenta (BUTERA, 1995).

\section{Conclusão}

Com os resultados das aplicações das regressões lineares e aplicações em campo se conclui que a metodologia é válida para a calibração de sensores diferentes em relação a um sensor de referência, podendo ser uma estratégia interessante para os pesquisadores, que não possuem diversos sensores calibrados.

\section{Referências}

BARATTO, J.; GALVANI, E.; WOLLMANN, C.A. Calibração e desempenho do abrigo meteorológico de baixo custo em condições de campo. Rev. Bras. Climatol., v.26, p.441-456, 2020. doi: 10.5380/abclima.v26i0.69834.

BUTERA, F.M. Architettura e ambiente: manuale per il controllo della qualità termica, luminosa e acustica degti edí, fici. Etsalibri Milano, 1995.

DA CUNHA, A.R.; ESCOBEDO, J.F.; GALVANI, E. Avaliação de um psicrômetro de termopar de baixo custo. Rev. Bras. Agrometeorol., v.9, n.1, p.17-23, 2001.

GARTLAND, L. Ilhas de calor: como mitigar zonas de calor em áreas urbanas. São Paulo: Oficina de Textos, 2011.

MACIEL, C.R. Análise da relação entre características do ambiente urbano e o comportamento de variáveis microclimáticas: estudo de caso em Cuiabá-MT. Cuiabá: Universidade Federal de Mato Grosso, 2014.

MAROCO, J. Análise estatística: com utilização do SPSS. 2007.

MARTINS, E. G. M. Coeficiente de correlação amostral. Revista de Ciência Elementar, v. 2, n. 2, p. 69, 2014. doi: 10.24927/ rce2018.024.

NOVAIS, J.W.Z. et al. Simulação por ENVI-met das Condições Higrotérmicas da Universidade de Cuiabá, Campus Barão. Rev. Ensino, Educ. Ciênc. Hum., v.21, n.2, p.200-205, 2020. doi: 10.17921/2447-8733.2020v21n2p200-205.

ROJAS, J; GILETE, S.D; MARZON, J. Development of a lowcost weather station to measure in situ essential climate variables. J. Earth Scie. Eng., n.4, p.455-463, 2014.

SOUSA, R.R.; ANTUNES, J.P.; CABRAL, I. Estação meteorológica experimental de baixo custo. Geo UERJ, n.27, p.80-97, 2015. doi: 10.12957/geouerj.2015.12335.

TORRES, J. D. et al. Aquisição de dados meteorológicos através da plataforma Arduino: construção baixo custo e análise de dados. Scie. Plena, v.11, n.2, p.1-13, 2015. 